\title{
SOME CONTROLLABILITY RESULTS FOR LINEAR VISCOELASTIC FLUIDS*
}

\author{
J.L. BOLDRINI ${ }^{\dagger}$, A. DOUBOVA ${ }^{\ddagger}$, E. FERNÁNDEZ-CARA ${ }^{\ddagger}$, AND M. GONZÁLEZ-BURGOS ${ }^{\ddagger}$
}

\begin{abstract}
We analyze the controllability properties of systems which provide a description, at first approximation, of a kind of viscoelastic fluid. We consider linear Maxwell fluids. First, we establish the large time approximate-finite dimensional controllability of the system, with distributed or boundary controls supported by arbitrary small sets. Then, we prove the large time exact controllability of fluids of the same kind with controls supported by suitable large sets. The proofs of these results rely on classical arguments. In particular, the approximate controllability result is implied by appropriate unique continuation properties, while exact controllability is a consequence of observability (inverse) inequalities. We also discuss questions concerning the controllability of viscoelastic fluids and some related open problems.
\end{abstract}

Key words. controllability, Maxwell fluids, vicoelastic fluids

AMS subject classifications. 93B05, 35B37, 76A10, 76D55, 93C20

DOI. $10.1137 / 100813592$

1. Introduction and main results. Let $\Omega \subset \mathbb{R}^{N}(N=2$ or 3$)$ be a bounded connected open set with boundary $\partial \Omega$ of class $C^{2}$ (for example). Let $\mathcal{O} \subset \Omega$ be a nonempty open set and let $\gamma \subset \partial \Omega$ be a nonempty relative open set such that the set $\partial \Omega \backslash \gamma$ has positive measure.

In this paper, we will sometimes (but not always) assume that

$$
\gamma \supset \Gamma\left(x^{0}\right):=\left\{x \in \partial \Omega:\left(x-x^{0}\right) \cdot \vec{n}(x)>0\right\}
$$

for some $x^{0} \in \mathbb{R}^{N}$, where $\vec{n}(x)$ is the outward unit normal vector to $\Omega$ at the point $x \in \partial \Omega$. We will also set

$$
\vec{m}(x) \equiv x-x^{0}, \quad R\left(x^{0}\right)=\max _{x \in \bar{\Omega}}|\vec{m}(x)|, \quad T\left(x^{0}\right)=2 R\left(x^{0}\right) .
$$

(We have used the notation introduced by J.-L. Lions in [15].) Similarly, we will sometimes consider open sets $\mathcal{O}$ satisfying

$$
\mathcal{O} \supset \mathcal{G}_{\delta}\left(x^{0}\right):=\bigcup_{x \in \Gamma\left(x^{0}\right)} B(x ; \delta) \cap \Omega
$$

for some $\delta>0$, where $B(x ; \delta)$ is the open ball centered at $x$ of radius $\delta$.

In fact, assumption (1) or (3) will be needed when dealing with exact controllability results, but not in the framework of approximate controllability; see below for the definition of these properties and the precise statements.

Let $T>0$ be a prescribed final time. In the paper, we will use the following notation: $Q=\Omega \times(0, T), \Sigma=\partial \Omega \times(0, T), \Sigma\left(x^{0}\right)=\Gamma\left(x^{0}\right) \times(0, T) ; \mathcal{L}_{S}\left(\mathbb{R}^{N}\right)$ will

\footnotetext{
${ }^{*}$ Received by the editors November 2, 2010; accepted for publication (in revised form) December 20, 2011; published electronically April 17, 2012. The authors were partially supported by DGES grant MTM2010-15592.

http://www.siam.org/journals/sicon/50-2/81359.html

${ }^{\dagger}$ IMECC-UNICAMP, C.P. 6065, 13081-970, Campinas-SP, Brazil (boldrini@ime.unicamp.br).

${ }_{\ddagger}^{\ddagger}$ Dpto. E.D.A.N., University of Sevilla, Aptdo. 1160, 41080 Sevilla, Spain (doubova@us.es, cara@ us.es, manoloburgos@us.es).
} 
denote the space of symmetric real $N \times N$-matrices; $|\cdot|$ and $\|\cdot\|($ resp., $(\cdot, \cdot)$ and $((\cdot, \cdot)))$ will stand for the usual norms (resp., scalar products) in $L^{2}(\Omega)$ and $H_{0}^{1}(\Omega)$; $\langle\cdot, \cdot\rangle$ will be used to denote several duality products; and $C$ will denote a generic positive constant.

The following spaces will be needed:

$$
\left\{\begin{array}{l}
\mathcal{V}=\left\{\phi \in C_{0}^{\infty}(\Omega)^{N}: \nabla \cdot \phi=0 \text { in } \Omega\right\} \\
H=\left\{w \in L^{2}(\Omega)^{N}: \nabla \cdot w=0 \text { in } \Omega, w \cdot n=0 \text { on } \partial \Omega\right\} \\
V=\left\{w \in H_{0}^{1}(\Omega)^{N}: \nabla \cdot w=0 \text { in } \Omega\right\} \\
\Phi=L^{2}\left(\Omega ; \mathcal{L}_{S}\left(\mathbb{R}^{N}\right)\right)
\end{array}\right.
$$

Moreover, for any $\tau_{0} \in \Phi$, we will set

$$
\Phi_{0}=\{D w: w \in V\} \quad \text { and } \quad \Phi^{*}\left(\tau_{0}\right)=e^{-a T} \tau_{0}+\Phi_{0},
$$

where $D y$ is the usual deformation tensor:

$$
D y=\frac{1}{2}\left(\nabla y+{ }^{t} \nabla y\right) .
$$

The spaces $H, V$, and $\Phi$ are Hilbert spaces for the norms $|\cdot|,\|\cdot\|$, and $|\cdot|$, respectively. On the other hand, $\mathcal{V}$ is dense in $H$ and $V$.

We will consider the following system, which describes at least at first approximation the behavior of a viscoelastic fluid of the Maxwell kind:

$$
\begin{cases}\partial_{t} y+\nabla \pi=\nabla \cdot \tau+v 1_{\mathcal{O}} & \text { in } Q, \\ \nabla \cdot y=0 & \text { in } Q, \\ \partial_{t} \tau+a \tau=2 b D y & \text { in } Q, \\ y=0 & \text { on } \Sigma, \\ y(0)=y_{0}, \quad \tau(0)=\tau_{0} & \text { in } \Omega .\end{cases}
$$

Here, $1_{\mathcal{O}}$ is the characteristic function of $\mathcal{O}$; the functions $y=y(x, t), \pi=\pi(x, t)$, and $\tau=\tau(x, t)$ are, respectively, the velocity field, the pressure distribution, and the elastic stress tensor of the fluid (of course, $y=\left\{y_{i}\right\}$ and $\tau=\left\{\tau_{i j}\right\}$ for some scalar functions $y_{i}$ and $\tau_{i j}$ with $\tau_{i j}=\tau_{j i}$ ). It is assumed that the mass density of the fluid is $\rho_{0} \equiv 1 . a$ and $b$ are positive constants, $y_{0} \in H$ and $\tau_{0} \in \Phi$. tively.

The constants $a$ and $b$ have the dimensions of (time) $)^{-1}$ and (velocity) $)^{2}$, respec-

We can obviously make (4) dimensionless with the help of suitable length, time, and velocity reference units. For instance, we can introduce

$$
L=\lambda_{1}^{-1 / 2}, \quad T_{0} \text { and } U=\lambda_{1}^{-1 / 2} T_{0}^{-1},
$$

where $\lambda_{1}$ is the first eigenvalue of the Dirichlet Laplacian in $\Omega$ and $T_{0}$ is a characteristic time of the flow. In that case, (4) is rewritten as a similar system where the new parameters $a$ and $b$ satisfy the following:

- $a=\mathrm{We}^{-1}$, the reciprocal of the Weissenberg number of the fluid. By definition, We is the ratio of the relaxation time and the characteristic time $T_{0}$ and physically represents the rate at which elastic energy is stored or released. In other words, We can be used as a "measure" of the elasticity of the media.

- $b=\mathrm{We}^{-1} \mathrm{Re}^{-1}$, where Re is the Reynolds number of the fluid, i.e.,

$$
\mathrm{Re}=\frac{U L}{\nu}=\frac{1}{\lambda_{1} T_{0} \nu},
$$

where $\nu$ is the kinematic viscosity. 
For more details on the physical meaning of these parameters, see, for instance, $[24$, $13]$.

In (4), $v \in L^{2}(\mathcal{O} \times(0, T))^{N}$ is a distributed control with support in the cylinder $\mathcal{O} \times(0, T)$. It can be interpreted as an external force field that is active in a part of the fluid domain. We will refer to (4) as the distributed controlled problem for a Maxwell fluid.

We will also consider similar boundary controlled problems, that is, systems of the form

$$
\begin{cases}\partial_{t} y+\nabla \pi=\nabla \cdot \tau & \text { in } Q \\ \nabla \cdot y=0 & \text { in } Q \\ \partial_{t} \tau+a \tau=2 b D y & \text { in } Q \\ y=1_{\gamma} r & \text { on } \Sigma \\ y(0)=y_{0}, \quad \tau(0)=\tau_{0} & \text { in } \Omega\end{cases}
$$

where $1_{\gamma}$ is the characteristic function of $\gamma$ and $r \in L^{2}(\gamma \times(0, T))^{N}$ is a boundary control, acting on $\gamma \times(0, T)$.

Notice that in this case, we must deal in principle with linear time-dependent equations where we prescribe some nonregular data on a part of the boundary. Nevertheless, we will be able to solve problems of this kind by introducing suitable auxiliary systems.

These models can be respectively viewed as first approximations (when $y$ and $\tau$ are small) of the following nonlinear systems (see [13]):

$$
\begin{cases}\partial_{t} y+(y \cdot \nabla) y+\nabla \pi=\nabla \cdot \tau+v 1_{\mathcal{O}} & \text { in } Q \\ \nabla \cdot y=0 & \text { in } Q \\ \partial_{t} \tau+(y \cdot \nabla) \tau+g(\nabla y, \tau)+a \tau=2 b D y & \text { in } Q \\ y=0 & \text { on } \Sigma \\ y(0)=y_{0}, \quad \tau(0)=\tau_{0} & \text { in } \Omega\end{cases}
$$

and

$$
\begin{cases}\partial_{t} y+(y \cdot \nabla) y+\nabla \pi=\nabla \cdot \tau & \text { in } Q \\ \nabla \cdot y=0 & \text { in } Q \\ \partial_{t} \tau+(y \cdot \nabla) \tau+g(\nabla y, \tau)+a \tau=2 b D y & \text { in } Q \\ y=1_{\gamma} r & \text { on } \Sigma \\ y(0)=y_{0}, \quad \tau(0)=\tau_{0} & \text { in } \Omega .\end{cases}
$$

Here, $(y \cdot \nabla) y$ and $(y \cdot \nabla) \tau$ are the usual transport terms and

$$
g(\nabla y, \tau)=\tau \cdot W y-W y \cdot \tau-\kappa(D y \cdot \tau+\tau \cdot D y),
$$

where $W y=\frac{1}{2}\left(\nabla y-{ }^{t} \nabla y\right)$ is the vorticity tensor and $\kappa \in[-1,1]$.

In this paper, we will analyze some controllability properties of the linear systems (4) and (5). This has already been the objective of some previous works; see [22, 19].

To this purpose, let us first see that after an appropriate change of variables, (4) and (5) can be equivalently rewritten as second-order in time hyperbolic systems.

More precisely, let $(y, \pi, \tau)$ be a solution to (4) and let us set

$$
\left\{\begin{array}{l}
z(t)=\int_{0}^{t} e^{a s} y(s) d s, \quad Z=e^{a t} \pi, \quad u=e^{a t} v, \text { and } \\
\tau(t)=e^{-a t} \tau_{0}+2 b \int_{0}^{t} e^{-a(t-s)} D y(s) d s \equiv e^{-a t} \tau_{0}+2 b e^{-a t} D z(t) .
\end{array}\right.
$$

Copyright (C) by SIAM. Unauthorized reproduction of this article is prohibited. 
From these identities, we easily see that $\tau$ satisfies the second equation of (4) and the initial condition $\tau(0)=\tau_{0}$ and also that

$$
\partial_{t} y+\nabla \pi=2 b e^{-a t} \Delta z+e^{-a t} \nabla \cdot \tau_{0}+v 1_{\mathcal{O}} .
$$

Multiplying by $e^{a t}$ the previous identity and taking into account that

$$
\partial_{t} z=e^{a t} y, \quad \partial_{t t} z=a \partial_{t} z+e^{a t} \partial_{t} y
$$

we deduce that $z$ solves, together with some $Z$, the linear problem

$$
\begin{cases}\partial_{t t} z-a \partial_{t} z-b \Delta z+\nabla Z=\nabla \cdot \tau_{0}+u 1_{\mathcal{O}} & \text { in } Q, \\ \nabla \cdot z=0 & \text { in } Q, \\ z=0 & \text { on } \Sigma, \\ z(0)=z^{0}, \quad \partial_{t} z(0)=z^{1} & \text { in } \Omega,\end{cases}
$$

where $\left(z^{0}, z^{1}\right)=\left(0, y_{0}\right)$.

A similar reformulation can be found for (5). Indeed, if $(y, \pi, \tau)$ solves (5), we conserve the definitions of $z$ and $Z$ in (8) and we set

$$
w=\int_{0}^{t} e^{a s} r(s) d s
$$

we also find that

$$
\begin{cases}\partial_{t t} z-a \partial_{t} z-b \Delta z+\nabla Z=\nabla \cdot \tau_{0} & \text { in } Q, \\ \nabla \cdot z=0 & \text { in } Q, \\ z=w 1_{\gamma} & \text { on } \Sigma, \\ z(0)=z^{0}, \quad \partial_{t} z(0)=z^{1} & \text { in } \Omega .\end{cases}
$$

From (8) and (10), it becomes clear that the existence, uniqueness, and controllability properties of (4) and (5) can be reformulated in terms of similar properties of (9) and (11), respectively.

Notice that in view of the expression of $\tau$ in (8), $\tau(T)$ belongs to the linear manifold $\Phi^{*}\left(\tau_{0}\right)$. Thus, in order to introduce good definitions of controllability, we will always fix the desired elastic stress tensor $\tau_{d}$ in $\Phi^{*}\left(\tau_{0}\right)$. For technical reasons, it will also be assumed that $\nabla \cdot \tau_{0} \in L^{2}(\Omega)^{N}$.

It will be said that (4) (resp., (5)) is approximately controllable in $H \times \Phi$ at time $T$ if for any $y_{0}, y_{d} \in H$, any $\tau_{0} \in \Phi$, any $\tau_{d} \in \Phi^{*}\left(\tau_{0}\right)$, and any $\varepsilon>0$, there exist distributed controls $v \in L^{2}(\mathcal{O} \times(0, T))^{N}$ (resp., boundary controls $r \in L^{2}(\gamma \times(0, T))^{N}$ ) such that the associated solutions satisfy

$$
\left|y(T)-y_{d}\right| \leq \varepsilon, \quad\left|\tau(T)-\tau_{d}\right| \leq \varepsilon .
$$

It will be said that (4) (resp., (5)) is approximately finite dimensional controllable if for any finite dimensional space

$$
Y \subset H \times \Phi_{0} \quad\left(\text { resp. }, \tilde{Y} \subset H \times \Phi_{0}\right),
$$

any initial and final (desired) data as above, and any $\varepsilon>0$, the previous controls $v$ and $r$ can be chosen such that in addition to (12), the following holds:

$$
P_{Y}(y(T), \tau(T))=P_{Y}\left(y_{d}, \tau_{d}\right) \quad\left(\operatorname{resp} ., P_{\tilde{Y}}(y(T), \tau(T))=P_{\tilde{Y}}\left(y_{d}, \tau_{d}\right)\right) .
$$

Here, $P_{Y}: H \times \Phi \mapsto Y$ and $P_{\tilde{Y}}: H \times \Phi \mapsto \tilde{Y}$ are the usual orthogonal projectors. 
It will be said that (4) (resp., (5)) are exactly controllable at time $T$ if for any $y_{0}, y_{d} \in H$, any $\tau_{0} \in \Phi$, and any $\tau_{d} \in \Phi^{*}\left(\tau_{0}\right)$, there exists $v \in L^{2}(\mathcal{O} \times(0, T))^{N}$ (resp., $\left.r \in L^{2}(\gamma \times(0, T))^{N}\right)$ such that the associated solutions satisfy

$$
y(T)=y_{d}, \quad \tau(T)=\tau_{d} .
$$

Finally, if this is satisfied for any $y_{0} \in H$, any $\tau_{0} \in \Phi$, and the desired states $y_{d}=0$ and $\tau_{d}=0$, it will be said that the considered system is null controllable at time $T$.

The controllability properties of systems (4) and (5) will be deduced from the properties of the solutions to the linear hyperbolic systems (9) and (11). The latter can be viewed as incompressible nonscalar versions of the wave equation. Consequently, it will be convenient to recall some known results on the exact and approximate controllability of the wave equation and, also, of systems of the Stokes kind.

For any nonempty $\mathcal{O}$ and $\gamma$ (not necessarily satisfying (1)), the approximate distributed and boundary controllability of the wave equation hold if $T$ is large enough; see [15].

In [11], it was proved that the boundary exact controllability result for the wave equation also holds if $\gamma \supset \Gamma\left(x^{0}\right)$ and $T>0$ is large enough. Later, this result was improved in [15], where the optimal control time $T\left(x^{0}\right)$ was found. These results were proved by the multipliers method, which relies on computing the global integral in $Q$ of the product of the equation by the gradient of the solution following some convenient vector field and then performing appropriate integrations by parts. Let us also mention the work [16], where a more general kind of boundary control sets was found. The distributed exact controllability of the wave equation was deduced in [15] from the boundary exact controllability if $\mathcal{O}$ is a neighborhood of $\Gamma\left(x^{0}\right)$ and $T>T\left(x^{0}\right)$.

In [1], by using microlocal analysis and geometric optic techniques, a necessary and sufficient condition and a minimal control time were obtained for the exact controllability of the wave equation in regular domains. We also refer to [18], [27], and the references therein for boundary and distributed exact controllability results for the wave equation with a potential. These last results were established with the help of global Carleman estimates.

The approximate controllability of the classical Stokes equations is well known. For general linear systems of the Stokes kind with distributed control, the same property was estalished in [6]. Some partial results concerning the approximate controllability for the Navier-Stokes system can be found, for example, in $[6,7,3,4]$. The null controllability of the Stokes and Navier-Stokes systems has been studied intensively in recent years. For instance, let us mention the papers [10, 12, 8, 9].

Our first main result concerns the approximate-finite dimensional controllability of (4).

THEOREM 1.1. There exists a positive time $T_{1}=T_{1}(b, \Omega, \mathcal{O})$ such that if $T>T_{1}$, (4) is approximately finite dimensional controllable at time $T$.

The proof relies on a unique continuation property for the solutions of a system similar to (9). We need a large time $T$ due to hyperbolicity. This property will be established in section 2.1 as a consequence of the classical Holmgren uniqueness theorem.

A consequence of Theorem 1.1 is that for large $T$, (5) is also approximately controllable.

THEOREM 1.2. There exists a positive time $\tilde{T}_{1}=\tilde{T}_{1}(b, \Omega, \gamma)$ such that if $T>\tilde{T}_{1}$, then (5) is approximately finite dimensional controllable at time $T$.

Copyright $@$ by SIAM. Unauthorized reproduction of this article is prohibited. 
The main tool for the proof of these two results is a uniqueness theorem that ultimately relies on Holmgren's principle. See [15, Chapter 1, section 8]. This provides unique continuation only for large times, depending on $b$ but independent of $a$.

Our third main result deals with the exact controllability of (4).

THEOREM 1.3. Let us assume that (3) is satisfied for some $x^{0} \in \mathbb{R}^{N}$ and some $\delta>0$ and

$$
0<a<2 \sqrt{\lambda_{1} b}
$$

where $\lambda_{1}$ is the first eigenvalue of the Dirichlet Laplacian in $\Omega$. There exists $T_{2}=$ $T_{2}\left(a, b, \Omega, x^{0}, \delta\right)$ with the following property: for all $T>T_{2}$, there exists a Hilbert space $F$ such that (4) is exactly controllable at time $T$ with initial and final data in $F^{\prime}$. More precisely, for all $\left(y_{0}, \tau_{0}\right),\left(y_{d}, \tau_{d}\right) \in F^{\prime}$ with $\tau_{d} \in \Phi^{*}\left(\tau_{0}\right)$, there exists $v \in L^{2}(\mathcal{O} \times(0, T))^{N}$ such that

$$
y(T)=y_{d}, \quad \tau(T)=\tau_{d} .
$$

Moreover, $F^{\prime}$ contains all couples $(y, \tau)$ with $y \in H$ and $\tau \in \Phi$.

Concerning the exact controllability of (5), we have the following.

THEOREM 1.4. Let us assume that (1) is satisfied for some $x^{0} \in \mathbb{R}^{N}$, $a$ and $b$ satisfy (15), and $T>\tilde{T}_{2}$, where

$$
\tilde{T}_{2}=\frac{T\left(x^{0}\right)}{\min \left(1, b-a^{2} /\left(4 \lambda_{1}\right)\right)}
$$

and $T\left(x^{0}\right)$ is given by (2). There exists a Hilbert space $\tilde{F}$ such that (5) is exactly controllable at time $T$ with initial and final data in $\tilde{F}^{\prime}$. Moreover, $\tilde{F}^{\prime}$ contains all couples $(y, \tau)$ with $y \in H$ and $\tau \in \Phi$.

The first key point of the proofs of these results will be a "boundary" observability inequality for a hyperbolic (adjoint) system similar to (11). The second key point will be a similar "distributed" estimate. Then, the proofs will be achieved by adapting the Hilbert uniqueness method (HUM), introduced by J.-L. Lions in [14] and [15].

Note. Notice that, in physical terms, taking into account that $a=\mathrm{We}^{-1} T_{0}^{-1}$ and $b=\mathrm{We}^{-1} \mathrm{Re}^{-1} \lambda_{1}^{-1} T_{0}^{-2},(15)$ can be rewritten as follows:

$$
\frac{\mathrm{Re}}{\mathrm{We}}<4
$$

If we denote by $T_{r}$ the relaxation time, then

$$
\mathrm{We}=\frac{T_{r}}{T_{0}}
$$

and the assumption (15) reads

$$
\operatorname{Re}^{*}:=\frac{1}{\lambda_{1} T_{r} \nu}<4
$$

This means that the "elastic Reynolds number" Re* cannot be large in Theorems 1.3 and 1.4. The extension of these results to the case of more general control sets $\mathcal{O}$ or $\gamma$ (for instance, as in [16]) or general positive $a$ and $b$ not necessarily satisfying (15) is an open question; see section 4 for more details.

The rest of this paper is organized as follows. 
In section 2, we will analyze the approximate-finite dimensional controllability of (4) and (5): we prove a unique continuation property and we give the proofs of the first two main results (Theorems 1.1 and 1.2). For these proofs, the main ideas have been taken from [15] and [28].

Section 3 is devoted to proving the exact controllability of (4) and (5), that is, Theorems 1.3 and 1.4. The key point is to deduce a suitable boundary inverse inequality that holds when $\gamma$ satisfies (1) and $T$ is large enough. To this end, we apply the multipliers method.

In section 4, we make some additional remarks and comments and we mention some open questions. Finally, the proof of a basic existence and uniqueness result is sketched in an appendix.

2. Proofs of Theorems 1.1 and 1.2. In this section, we consider the controlled linear Maxwell systems (4) and (5).

Recall that thanks to the change of variables (8), (4) is equivalent to (9) with $\left(z^{0}, z^{1}\right)=\left(0, y_{0}\right)$. Accordingly, we will first prove that (9) is approximately finite dimensional controllable, and we will then deduce Theorem 1.1 from this.

Theorem 1.2 will be a straightforward consequence of this result.

We will denote by $A$ the usual Stokes operator in $\Omega$. Recall that $A: D(A) \mapsto H$ with

$$
D(A)=H^{2}(\Omega)^{N} \cap V, \quad A w=P_{H}(-\Delta w) \quad \forall w \in D(A),
$$

where $P_{H}: L^{2}(\Omega)^{N} \mapsto H$ is the orthogonal projector.

We will begin by considering a slightly more general problem:

$$
\begin{cases}\partial_{t t} z-a \partial_{t} z-b \Delta z+c z+\nabla Z=f & \text { in } Q \\ \nabla \cdot z=0 & \text { in } Q \\ z=0 & \text { on } \Sigma \\ z(0)=z^{0}, \quad \partial_{t} z(0)=z^{1} & \text { in } \Omega\end{cases}
$$

where $a, c \in \mathbb{R}, b>0$, and $z^{0}$ and $z^{1}$ are given.

We have the following existence, uniqueness, and regularity result.

TheOREm 2.1. Let us first assume that $f=0$. For any $\left(z^{0}, z^{1}\right) \in H \times V^{\prime}$, there exists a unique solution $(z, Z)$ to $(16)$ ( $Z$ is unique up to a distribution in $\left.W^{-1, \infty}(0, T)\right)$ that satisfies

$$
z \in C^{0}([0, T] ; H) \cap C^{1}\left([0, T] ; V^{\prime}\right) \cap C^{2}\left([0, T] ; D(A)^{\prime}\right)
$$

If $\left(z^{0}, z^{1}\right) \in V \times H$, one also has

$$
z \in C^{0}([0, T] ; V) \cap C^{1}([0, T] ; H) \cap C^{2}\left([0, T] ; V^{\prime}\right)
$$

and the pressure $Z$ can be chosen in $L^{2}\left(0, T ; L^{2}(\Omega)\right)$. Moreover, if $\left(z^{0}, z^{1}\right) \in D(A) \times V$, then

$$
z \in C^{0}([0, T] ; D(A)) \cap C^{1}([0, T] ; V) \cap C^{2}([0, T] ; H)
$$

holds, and $Z$ can be chosen in $C^{0}\left([0, T] ; H^{1}(\Omega)\right)$.

Let us now consider nonzero data $f$ in (16). If $f \in L^{1}\left(0, T ; H^{-1}(\Omega)^{N}\right)$ and $\left(z^{0}, z^{1}\right) \in H \times V^{\prime}$, there exists a unique "generalized" solution to (16) with $z \in$ $C^{0}([0, T] ; H) \cap C^{1}\left([0, T] ; V^{\prime}\right)$. That solution is "classical," that is, $z \in C^{0}([0, T] ; V) \cap$ 
$C^{1}([0, T] ; H) \cap C^{2}\left([0, T] ; V^{\prime}\right)$, if one additionally has $\partial_{t} f \in L^{1}\left(0, T ; H^{-1}(\Omega)^{N}\right)$ and $\left(z^{0}, z^{1}\right) \in V \times H$.

If $f \in L^{1}(0, T ; H)$ and $\left(z^{0}, z^{1}\right) \in V \times H$, the solution to (16) satisfies $z \in$ $C^{0}([0, T] ; V) \cap C^{1}([0, T] ; H)$; if $\partial_{t} f \in L^{1}(0, T ; H)$ and $\left(z^{0}, z^{1}\right) \in D(A) \times V$, then (16) satisfies $z \in C^{0}([0, T] ; D(A)) \cap C^{1}([0, T] ; V) \cap C^{2}([0, T] ; H)$.

Finally, all these assertions hold with continuous dependence with respect to the data. Thus, for instance, if $f=0$ and $\left(z^{0}, z^{1}\right) \in V \times H$, the solution to (16) satisfies

$$
\left\{\begin{array}{c}
\|z\|_{C^{0}([0, T] ; V)}+\left\|\partial_{t} z\right\|_{C^{0}([0, T] ; H)}+\left\|\partial_{t t} z\right\|_{C^{2}\left([0, T] ; V^{\prime}\right)}+\|Z\|_{L^{2}\left(0, T ; L^{2}\right)} \\
\quad \leq C\left\|\left(z^{0}, z^{1}\right)\right\|_{V \times H}
\end{array}\right.
$$

for some positive $C$ only depending on $a, b, c, \Omega$, and $T$.

This result can be proved using classical and very well known arguments from semigroup theory; for completeness, we provide a sketch of the proof in the appendix.

Notice that due to the time reversibility of (16), Theorem 2.1 also holds if in (16) the initial conditions are replaced by the final conditions

$$
z(T)=z^{0}, \quad \partial_{t} z(T)=z^{1} \quad \text { in } \quad \Omega .
$$

Taking $c=0$ and using (8), we easily deduce the following.

TheOrem 2.2. Let us assume that $y_{0} \in H, \tau_{0} \in \Phi$, and $v \in L^{2}(\mathcal{O} \times(0, T))^{N}$. Then there exists a unique solution $(y, \pi, \tau)$ to (4) with

$$
y \in C^{0}([0, T] ; H), \quad \partial_{t} y \in C^{0}\left([0, T] ; V^{\prime}\right), \quad \tau \in C^{0}([0, T] ; \Phi) .
$$

Furthermore, if one also has $y_{0} \in V, \nabla \cdot \tau_{0} \in L^{2}(\Omega)^{N}$, and $\partial_{t}\left(1_{\mathcal{O}} v\right) \in L^{2}(\mathcal{O} \times(0, T))^{N}$, then

$$
y \in C^{0}([0, T] ; V), \quad \partial_{t} y \in C^{0}([0, T] ; H), \quad \tau \in C^{1}([0, T] ; \Phi) .
$$

2.1. A first unique continuation property. In this section, we will present a unique continuation property which will be crucial in the proof of Theorem 1.1. Our starting point is the following result, which is proved in [15] as a consequence of Holmgren's principle (see, for instance, [2]).

Lemma 2.3. There exists a positive time $T_{1}=T_{1}(b, \Omega, \mathcal{O})$ such that if $T>T_{1}$, the following holds: If $h \in L^{\infty}\left(0, T ; H^{1}(\Omega)\right), \partial_{t} h \in L^{\infty}\left(0, T ; L^{2}(\Omega)\right)$,

$$
\begin{cases}\partial_{t t} h-a \partial_{t} h-b \Delta h=0 & \text { in } Q, \\ h=0 & \text { on } \Sigma,\end{cases}
$$

and

$$
h=0 \quad \text { on } \mathcal{O} \times(0, T)
$$

then $h \equiv 0$.

Notice that if $h$ has the regularity in this lemma and satisfies (17), one actually has $h \in C^{0}\left([0, T] ; H_{0}^{1}(\Omega)\right)$ and $\partial_{t} h \in C^{0}\left([0, T] ; L^{2}(\Omega)\right)$.

Lemma 2.4. Let us assume that $T>T_{1}$, where $T_{1}$ is furnished by Lemma 2.3 . Then the following holds: If $\varphi \in L^{\infty}(0, T ; V), \partial_{t} \varphi \in L^{\infty}(0, T ; H), p \in \mathcal{D}^{\prime}(Q)$,

$$
\begin{cases}\partial_{t t} \varphi-a \partial_{t} \varphi-b \Delta \varphi+\nabla p=0 & \text { in } Q \\ \nabla \cdot \varphi=0 & \text { in } Q \\ \varphi=0 & \text { on } \Sigma\end{cases}
$$

Copyright $@$ ( ) by SIAM. Unauthorized reproduction of this article is prohibited. 
and

$$
\varphi=0 \quad \text { in } \mathcal{O} \times(0, T)
$$

then $\varphi \equiv 0$.

Proof. Thanks to (19), we deduce from the first equation of (18) that

$$
\nabla p=0 \text { in } \mathcal{O} \times(0, T) .
$$

Therefore, $p$ is space-independent in $\mathcal{O} \times(0, T)$. On the other hand, applying the divergence operator to the first equation of (18) and using that $\nabla \cdot \varphi=0$, we obtain

$$
\Delta p=0 \quad \text { in } \quad Q .
$$

Consequently,

$$
\nabla p=0 \quad \text { in } \quad Q
$$

and $p$ is also space-independent in $Q$.

The system satisfied by $\varphi$ is thus reduced to $N$ uncoupled scalar equations. By applying Lemma 2.3 to the components of $\varphi$, we find that $\varphi \equiv 0$.

This ends the proof.

As we said in section 1 , the time $T_{1}$ provided by Lemma 2.3 is independent of $a$. This is clearly seen in the arguments in [15], where only the principal part of the PDE in (17) is relevant.

2.2. Approximate-finite dimensional controllability. This section is devoted to proving Theorems 1.1 and 1.2 .

The proof of Theorem 1.1 will be a consequence of the following result.

Proposition 2.5. Let us assume that $T>T_{1}$, where $T_{1}=T_{1}(b, \Omega, \mathcal{O})$ is furnished by Lemma 2.3. Then, the approximate-finite dimensional controllability of (9) holds.

In other words, for any $\tau_{0} \in \Phi$, any $\left(z^{0}, z^{1}\right),\left(w^{0}, w^{1}\right) \in V \times H$, any finite dimensional space $E \subset V \times H$, and any $\varepsilon>0$, there exists $u \in L^{2}(\mathcal{O} \times(0, T))^{N}$ such that the associated solution of (9) satisfies

$$
\left\|\left(z(T), \partial_{t} z(T)\right)-\left(w^{0}, w^{1}\right)\right\|_{V \times H} \leq \varepsilon
$$

and

$$
P_{E}\left(z(T), \partial_{t} z(T)\right)=P_{E}\left(w^{0}, w^{1}\right),
$$

where $P_{E}: V \times H \mapsto E$ is the usual orthogonal projector.

Proof. We will write the solution to (9) in the form

$$
z=\eta+\hat{z}
$$

where $\eta \in C^{0}([0, T] ; V) \cap C^{1}([0, T] ; H)$ is (together with some pressure) the solution to (9) with $u \equiv 0$ (the uncontrolled solution) and $\hat{z}$ is (together with $\hat{Z}$ ) the solution to

$$
\begin{cases}\partial_{t t} \hat{z}-a \partial_{t} \hat{z}-b \Delta \hat{z}+\nabla \hat{Z}=u 1_{\mathcal{O}} & \text { in } Q, \\ \nabla \cdot \hat{z}=0 & \text { in } Q, \\ \hat{z}=0 & \text { on } \Sigma, \\ \hat{z}(0)=0, \quad \partial_{t} \hat{z}(0)=0 & \text { in } \Omega .\end{cases}
$$


The proof of Proposition 2.5 is then reduced to see that for any $\tau_{0},\left(z^{0}, z^{1}\right)$, $\left(w^{0}, w^{1}\right)$, and $E$ and $\varepsilon>0$, there exists a control $u \in L^{2}(\mathcal{O} \times(0, T))^{N}$ such that the solution to (22) satisfies

$$
\left\|\left(\hat{z}(T), \partial_{t} \hat{z}(T)\right)-\left(\hat{w}^{0}, \hat{w}^{1}\right)\right\|_{V \times H} \leq \varepsilon, \quad P_{E}\left(\hat{z}(T), \partial_{t} \hat{z}(T)\right)=P_{E}\left(\hat{w}^{0}, \hat{w}^{1}\right),
$$

where $\left(\hat{w}^{0}, \hat{w}^{1}\right)=\left(w^{0}-\eta(T), w^{1}-\partial_{t} \hat{\eta}(T)\right)$.

To this end, we will adapt the arguments in [28]. Thus, for any $\left(\varphi^{0}, \varphi^{1}\right) \in H \times V^{\prime}$, let us consider the adjoint system

$$
\begin{cases}\partial_{t t} \varphi+a \partial_{t} \varphi-b \Delta \varphi+\nabla p=0 & \text { in } Q \\ \nabla \cdot \varphi=0 & \text { in } Q \\ \varphi=0 & \text { on } \Sigma \\ \varphi(T)=\varphi^{0}, \quad \partial_{t} \varphi(T)=\varphi^{1} & \text { in } \Omega\end{cases}
$$

We know that (24) possesses a unique solution $(\varphi, p)$ ( $p$ is unique up to a distribution in $\left.W^{-1, \infty}(0, T)\right)$ satisfying $\varphi \in C^{0}([0, T] ; H)$ and $\partial_{t} \varphi \in C^{0}\left([0, T] ; V^{\prime}\right)$. Let us introduce the functional

$$
\left\{\begin{aligned}
J\left(\varphi^{0}, \varphi^{1}\right)= & \frac{1}{2} \iint_{\mathcal{O} \times(0, T)}|\varphi|^{2}+\varepsilon\left\|\left(I-P_{E}\right)\left(A_{0}^{-1} \varphi^{1}, \varphi^{0}\right)\right\|_{V \times H} \\
& -\left\langle\left(\varphi^{0}, \varphi^{1}\right),\left(\hat{w}^{1}-a \hat{w}^{0},-\hat{w}^{0}\right)\right\rangle \quad \forall\left(\varphi^{0}, \varphi^{1}\right) \in H \times V^{\prime},
\end{aligned}\right.
$$

where $\langle\cdot, \cdot\rangle$ denotes the duality pairing for $H \times V^{\prime}$ and $H \times V$ and $A_{0}: V \mapsto V^{\prime}$ is the usual isomorphism induced by the Stokes operator in $\Omega$. Notice that since $E$ is a finite dimensional space, the operator $P_{E}$ is compact.

From the unique continuation property in Lemma 2.4 and the compactness of $P_{E}$, it can be deduced, arguing as in the proof of Theorem 1.3 of [28], that $J: H \times V^{\prime} \mapsto \mathbb{R}$ is continuous and strictly convex and, moreover,

$$
\liminf _{\left\|\left(\varphi^{0}, \varphi^{1}\right)\right\|_{H \times V^{\prime}}} \frac{J\left(\varphi^{0}, \varphi^{1}\right)}{\left\|\left(\varphi^{0}, \varphi^{1}\right)\right\|_{H \times V^{\prime}}} \geq \varepsilon .
$$

Therefore, $J$ possesses exactly one minimizer $\left(\hat{\varphi}^{0}, \hat{\varphi}^{1}\right)$. Let us check that the control $u=\left.\hat{\varphi}\right|_{\mathcal{O} \times(0, T)}$, where $(\hat{\varphi}, \hat{p})$ solves $(24)$ with $\left(\hat{\varphi}^{0}, \hat{\varphi}^{1}\right)$ as final data, is such that the solution to $(22)$ satisfies $(23)$.

Indeed, for any $\left(\psi^{0}, \psi^{1}\right) \in H \times V^{\prime}$ and any $\lambda \in \mathbb{R}$, we have

$$
J\left(\hat{\varphi}^{0}, \hat{\varphi}^{1}\right) \leq J\left(\hat{\varphi}^{0}+\lambda \psi^{0}, \hat{\varphi}^{1}+\lambda \psi^{1}\right) .
$$

From this inequality, it is immediate that

$$
\begin{aligned}
0 \leq & \lambda \iint_{\mathcal{O} \times(0, T)} \hat{\varphi} \psi+\frac{\lambda^{2}}{2} \iint_{\mathcal{O} \times(0, T)}|\psi|^{2} \\
& +\varepsilon|\lambda|\left\|\left(I-P_{E}\right)\left(A_{0}^{-1} \psi^{1}, \psi^{0}\right)\right\|_{V \times H}-\lambda\left\langle\left(\psi^{0}, \psi^{1}\right),\left(\hat{w}^{1}-a \hat{w}^{0},-\hat{w}^{0}\right)\right\rangle .
\end{aligned}
$$

If we now take $\lambda>0$, divide by $\lambda$, and take limits as $\lambda \rightarrow 0^{+}$, we get

$$
\iint_{\mathcal{O} \times(0, T)} \hat{\varphi} \psi-\left\langle\left(\psi^{0}, \psi^{1}\right),\left(\hat{w}^{1}-a \hat{w}^{0},-\hat{w}^{0}\right)\right\rangle \geq-\varepsilon\left\|\left(I-P_{E}\right)\left(A_{0}^{-1} \psi^{1}, \psi^{0}\right)\right\|_{V \times H} .
$$

This, together with the same compuations for $\lambda<0$, leads to the following:

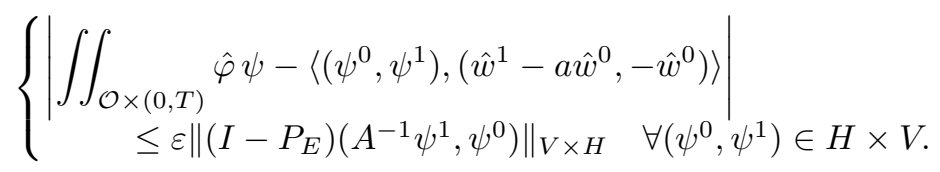

Copyright $@$ by SIAM. Unauthorized reproduction of this article is prohibited. 
On the other hand, if we multiply by $\psi$ the equation satisfied by $\hat{z}$ (see (22)) and we integrate by parts in $Q$, we see that

$$
\iint_{\mathcal{O} \times(0, T)} \hat{\varphi} \psi=\left\langle\left(\psi^{0}, \psi^{1}\right),\left(\partial_{t} \hat{z}(T)-a \hat{z}(T),-\hat{z}(T)\right)\right\rangle
$$

Taking into account this identity in (25) we deduce that

$$
\left\{\begin{array}{c}
\left|\left\langle\left(\psi^{0}, \psi^{1}\right),\left(\partial_{t} \hat{z}(T)-a \hat{z}(T),-\hat{z}(T)\right)\right\rangle-\left\langle\left(\psi^{0}, \psi^{1}\right),\left(\hat{w}^{1}-a \hat{w}^{0},-\hat{w}^{0}\right)\right\rangle\right| \\
\leq \varepsilon\left\|\left(I-P_{E}\right)\left(A_{0}^{-1} \psi^{1}, \psi^{0}\right)\right\|_{V \times H} \quad \forall\left(\psi^{0}, \psi^{1}\right) \in H \times V
\end{array}\right.
$$

which is clearly equivalent to (20)-(21).

We can now achieve the proof of Theorem 1.1.

Proof. Let us fix $y_{0}, y_{d} \in H, \tau_{0} \in \Phi, \tau_{d} \in \Phi^{*}\left(\tau_{0}\right)$, a finite dimensional space $Y \subset H \times \Phi$, and a real number $\varepsilon>0$.

First, notice that

$$
\|w\|^{2} \equiv \int_{\Omega}|\nabla w|^{2} d x=2 \int_{\Omega}|D w|^{2} d x \quad \forall w \in V .
$$

As a consequence, $\Phi_{0}$ is a closed subspace of $\Phi$ (and thus $\Phi^{*}\left(\tau_{0}\right)$ is a closed affine subspace of $\Phi)$ and $D: V \mapsto \Phi_{0}$ and $B:\left(z_{1}, z_{2}\right) \in V \times H \mapsto\left(z_{2}, D z_{1}\right) \in H \times \Phi_{0}$ are linear, bijective, and isometric.

Let $w_{d} \in V$ be such that $\tau_{d}=e^{-a T} \tau_{0}+w_{d}$ and let us set $\left(w^{0}, w^{1}\right)=\left(\frac{1}{2 b} e^{a T} w_{d}\right.$, $\left.e^{a T} y_{d}\right)$ and $E=B^{-1} Y$. By applying Proposition 2.5 to (9) for this $\left(w^{0}, w^{1}\right)$ and recalling again the change of variables (8), we deduce that there exists $v \in L^{2}(\mathcal{O} \times$ $(0, T))^{N}$ such that the unique solution to (4) satisfies (12) and (13).

This ends the proof.

Let us now give the proof of Theorem 1.2.

Proof. Let $y_{0}, y_{d}, \tau_{0}, \tau_{d}, \tilde{Y}$, and $\varepsilon$ be given and let us consider the boundary controlled system (5).

Let us introduce an auxiliary distributed controllability problem as follows.

Let $D$ be a new regular bounded domain with $D \supset \Omega, D \neq \Omega$, such that the restriction to $\Omega$ of any function $z \in H_{0}^{1}(D)$ satisfies

$$
z=0 \text { on } \partial \Omega \backslash \gamma
$$

Let us denote by $H(D), V(D)$, and $\Phi(D)$ the spaces $H, V$, and $\Phi$ relative to $D$. We will use that the extension by zero to $D$ of any $w \in H$ (resp., any $w \in V$, any $\sigma \in \Phi$ ) belongs to $H(D)$ (resp., $V(D), \Phi(D)$ ); this is obvious and well known.

Let $\hat{y}_{0}, \hat{y}_{d}, \hat{\tau}_{0}, \hat{\tau}_{d}$, and $\hat{Y}$ by the extensions by zero of $y_{0}, y_{d}, \tau_{0}, \tau_{d}$, and $\tilde{Y}$, respectively. We then have $\hat{y}_{0}, \hat{y}_{d} \in H(D), \hat{\tau}_{0}, \hat{\tau}_{d} \in \Phi(D), \hat{\tau}_{d}=e^{-a T} \hat{\tau}_{0}+D \hat{w}$ for some $\hat{w} \in V(D)$ and $\hat{Y} \subset H(D)$.

We can now choose an open set $\hat{\mathcal{O}} \subset D \backslash \bar{\Omega}$ and consider the system

$$
\begin{cases}\partial_{t} \hat{y}+\nabla \hat{\pi}=\nabla \cdot \hat{\tau}+\hat{v} 1_{\hat{\mathcal{O}}} & \text { in } \hat{Q} \\ \nabla \cdot \hat{y}=0 & \text { in } \hat{Q} \\ \partial_{t} \hat{\tau}+a \hat{\tau}=2 b D \hat{y} & \text { in } \hat{Q} \\ \hat{y}=0 & \text { on } \hat{\Sigma} \\ \hat{y}(0)=\hat{y}_{0}, \quad \hat{\tau}(0)=\hat{\tau}_{0} & \text { in } D\end{cases}
$$

where we have used the notation $\hat{Q}=D \times(0, T)$ and $\hat{\Sigma}=\partial D \times(0, T)$. 
From Theorem 1.1, we know that there exist $L^{2}$ controls $\hat{v}$ such that the corresponding states satisfy

$$
\left|(\hat{y}(T), \hat{\tau}(T))-\left(\hat{y}_{d}, \hat{\tau}_{d}\right)\right| \leq \varepsilon, \quad P_{\hat{Y}}(\hat{y}(T), \hat{\tau}(T))=P_{\hat{Y}}\left(\hat{y}_{d}, \hat{\tau}_{d}\right) .
$$

Now, if $y, \pi$, and $\tau$, respectively, denote the restrictions of $\hat{y}, \hat{\pi}$, and $\hat{\tau}$ to $\Omega \times(0, T)$, it is clear that they satisfy (5) for some $r \in L^{2}(\gamma \times(0, T))^{N}$ and also the desired controllability requirements.

This shows that (5) is approximately finite dimensional controllable at time $T$ and ends the proof.

3. Proofs of Theorems 1.3 and 1.4. This section is devoted to proving exact controllability results for (4) and (5). To this end, we will first establish some observability (inverse) estimates for the solutions to the linear system (24).

3.1. Some inverse inequalities. The first result of this section is the following boundary inverse inequality.

LEMma 3.1. Let $\gamma, a, b$, and $T$ satisfy the assumptions of Theorem 1.4. There exists a positive constant $C$, only depending on $a, b, \Omega, x^{0}$, and $T$, such that

$$
\left\|\left(\varphi^{0}, \varphi^{1}\right)\right\|_{V \times H}^{2} \leq C \iint_{\gamma \times(0, T)}\left|\frac{\partial \varphi}{\partial n}\right|^{2} d \sigma d t
$$

for any $\left(\varphi^{0}, \varphi^{1}\right) \in D(A) \times V$, where $\varphi$ is, together with some $p$, the associated solution to $(24)$.

Proof. Let us first perform a new change of variables in (24):

$$
\psi=e^{-a t / 2} \varphi, \quad q=e^{-a t / 2} p .
$$

Then (24) can be rewritten equivalently as follows:

$$
\begin{cases}\psi_{t t}-b \Delta \psi-\frac{a^{2}}{4} \psi+\nabla q=0 & \text { in } Q, \\ \nabla \cdot \psi=0 & \text { in } Q, \\ \psi=0 & \text { on } \Sigma, \\ \psi(T)=\psi^{0}, \quad \psi_{t}(T)=\psi^{1} & \text { in } \Omega .\end{cases}
$$

Lemma 3.1 will be proved if for some $C$ only depending on $a, b, \Omega, x^{0}$, and $T$, we establish the following inverse inequality for the solutions to (29):

$$
\left\|\left(\psi^{0}, \psi^{1}\right)\right\|_{V \times H}^{2} \leq C \iint_{\gamma \times(0, T)}\left|\frac{\partial \psi}{\partial n}\right|^{2} d \sigma d t
$$

for all $\left(\psi^{0}, \psi^{1}\right) \in D(A) \times V$. To this purpose, we will use the multipliers method, adapting arguments from $[14,15]$.

From Theorem 2.1, we deduce that the solution to $(29)$ satisfies $(\psi, q) \in C^{0}([0, T]$; $D(A)) \times L^{2}\left(0, T ; H^{1}(\Omega)\right)$. By multiplying the first equality in $(29)$ by $(\vec{m} \cdot \nabla) \psi$ (where $\vec{m}=\vec{m}(x)$ is given in (2)) and integrating in $Q$, we get

$$
\begin{aligned}
\iint_{Q} \partial_{t t} \psi \cdot m_{k} & \frac{\partial \psi}{\partial x_{k}}-b \iint_{Q} \Delta \varphi \cdot m_{k} \frac{\partial \psi}{\partial x_{k}} \\
- & \frac{a^{2}}{4} \iint_{Q} m_{k} \frac{\partial \psi}{\partial x_{k}} \psi+\iint_{Q} \nabla q \cdot m_{k} \frac{\partial \psi}{\partial x_{k}}=0,
\end{aligned}
$$

where we have used the standard convention of repeated index summation. 
After some integrations by parts, we see that the first term in the left-hand side of (30) is equal to

$$
\iint_{Q} \partial_{t t} \psi \cdot m_{k} \frac{\partial \psi}{\partial x_{k}}=\frac{1}{2} \iint_{Q} \frac{\partial m_{k}}{\partial x_{k}}\left|\partial_{t} \psi\right|^{2}+\left.\int_{\Omega} \partial_{t} \psi \cdot m_{k} \frac{\partial \psi}{\partial x_{k}}\right|_{t=0} ^{t=T} .
$$

The second term in the left-hand side can be written in the form

$$
\begin{aligned}
b \iint_{Q} \Delta \psi \cdot m_{k} \frac{\partial \psi}{\partial x_{k}}= & -b \iint_{Q} \frac{\partial \psi}{\partial x_{j}} \cdot\left(\frac{\partial m_{k}}{\partial x_{j}} \frac{\partial \psi}{\partial x_{k}}+m_{k} \frac{\partial^{2} \psi}{\partial x_{j} \partial x_{k}}\right) \\
& +b \iint_{\Sigma} \frac{\partial \psi}{\partial n} \cdot m_{k} \frac{\partial \psi}{\partial x_{k}} d \sigma d t
\end{aligned}
$$

Integrating by parts, we also obtain that

$$
\begin{aligned}
& b \iint_{Q} \frac{\partial \psi}{\partial x_{j}} \cdot m_{k} \frac{\partial^{2} \psi}{\partial x_{j} \partial x_{k}} \\
& \quad=-\frac{b}{2} \iint_{Q} \frac{\partial m_{k}}{\partial x_{k}}|\nabla \psi|^{2}+\frac{b}{2} \iint_{\Sigma} m_{k} n_{k}|\nabla \psi|^{2} d \sigma d t
\end{aligned}
$$

Using that $\psi=0$ on $\Sigma$, we deduce that

$$
|\nabla \psi|^{2}=\left|\frac{\partial \psi}{\partial n}\right|^{2} \quad \text { on } \quad \Sigma .
$$

Thus, from (30) the following identity is found:

$$
\begin{gathered}
\left.\int_{\Omega} \partial_{t} \psi \cdot m_{k} \frac{\partial \psi}{\partial x_{k}}\right|_{t=0} ^{t=T}+\frac{1}{2} \iint_{Q} \frac{\partial m_{k}}{\partial x_{k}}\left(\left|\partial_{t} \psi\right|^{2}-b|\nabla \psi|^{2}+\frac{a^{2}}{4}|\psi|^{2}\right) \\
\quad+b \iint_{Q} \frac{\partial m_{k}}{\partial x_{j}} \frac{\partial \psi}{\partial x_{j}} \cdot \frac{\partial \psi}{\partial x_{k}}-\frac{b}{2} \iint_{\Sigma} m_{k} n_{k}\left|\frac{\partial \psi}{\partial n}\right|^{2} d \sigma d t \\
=\iint_{Q}(-\nabla q) \cdot m_{k} \frac{\partial \psi}{\partial x_{k}} .
\end{gathered}
$$

Let us deduce the inverse inequality (27) from (31).

It is not difficult to see that

$$
\iint_{Q}(-\nabla q) \cdot m_{k} \frac{\partial \psi}{\partial x_{k}}=0
$$

Indeed, integrating by parts and taking into account that $\nabla \cdot \psi=0$, we get

$$
\iint_{Q}(-\nabla q) \cdot m_{k} \frac{\partial \psi}{\partial x_{k}}=\iint_{Q} q \frac{\partial m_{k}}{\partial x_{i}} \frac{\partial \psi_{i}}{\partial x_{k}}-\iint_{\Sigma} q m_{k} \frac{\partial \psi_{i}}{\partial x_{k}} n_{i} d \sigma d t
$$

The two integrals in the right-hand side of this equality vanish: the first one vanishes because $\frac{\partial m_{k}}{\partial x_{i}}=\delta_{i k}$ for $1 \leq i, k \leq N$ and $\nabla \cdot \psi=0$; the second one is zero because $\psi=0$ on $\Sigma$ and thus

$$
\frac{\partial \psi_{i}}{\partial x_{k}} n_{i}=\frac{\partial \psi_{i}}{\partial n} n_{i} n_{k}=\frac{\partial \psi_{i}}{\partial x_{i}} n_{k} \equiv 0
$$

Copyright (c) by SIAM. Unauthorized reproduction of this article is prohibited. 
Consequently, thanks to the choice of the function $\vec{m},(31)$ can be rewritten as follows:

$$
\begin{gathered}
\left.\int_{\Omega} \partial_{t} \psi \cdot m_{k} \frac{\partial \psi}{\partial x_{k}}\right|_{t=0} ^{t=T}+\frac{N}{2} \iint_{Q}\left(\left|\partial_{t} \psi\right|^{2}-b|\nabla \psi|^{2}+\frac{a^{2}}{4}|\psi|^{2}\right) \\
+b \iint_{Q}|\nabla \psi|^{2}-\frac{b}{2} \iint_{\Sigma} m_{k} n_{k}\left|\frac{\partial \psi}{\partial n}\right|^{2} d \sigma d t=0 .
\end{gathered}
$$

Notice that by multiplying by $\psi$ the first equation in (29) and integrating by parts in $Q$, we have

$$
A_{1}:=\iint_{Q}\left(\left|\partial_{t} \psi\right|^{2}-b|\nabla \psi|^{2}+\frac{a^{2}}{4}|\psi|^{2}\right)=\left.\int_{\Omega} \partial_{t} \psi \cdot \psi\right|_{t=0} ^{t=T}
$$

Let us also introduce

$$
A_{2}:=\left.\int_{\Omega} \partial_{t} \psi \cdot m_{k} \frac{\partial \psi}{\partial x_{k}}\right|_{t=0} ^{t=T}
$$

and let us denote by $E(t)$ the total energy of $\psi$ at time $t$, that is,

$$
E(t):=\frac{1}{2} \int_{\Omega}\left(\left|\partial_{t} \psi(t)\right|^{2}+|\nabla \psi(t)|^{2}-\frac{a^{2}}{4}|\psi(t)|^{2}\right) .
$$

Then, it is immediate that

$$
E(0)=E_{0}:=\frac{1}{2} \int_{\Omega}\left(\left|\psi^{1}\right|^{2}+b\left|\nabla \psi^{0}\right|^{2}-\frac{a^{2}}{4}\left|\psi^{0}\right|^{2}\right)
$$

and

$$
E(t)=E_{0} \quad \forall t \in[0, T]
$$

Taking into account this equality and the definitions of $A_{1}$ and $A_{2}$, we deduce from (32) that

$$
A_{2}+\frac{N-1}{2} A_{1}+T E_{0}+\frac{a^{2}}{4} \iint_{Q}|\psi|^{2}=\frac{b}{2} \iint_{\Sigma} m_{k} n_{k}\left|\frac{\partial \psi}{\partial n}\right|^{2} d \sigma d t .
$$

Since $\vec{m} \cdot \vec{n} \leq 0$ on $\Sigma \backslash \Sigma\left(x^{0}\right)$ and $|\vec{m} \cdot \vec{n}| \leq R\left(x^{0}\right)$ on $\Sigma\left(x^{0}\right)$, this gives

$$
A_{2}+\frac{N-1}{2} A_{1}+T E_{0}+\frac{a^{2}}{4} \iint_{Q}|\psi|^{2} \leq \frac{b R\left(x^{0}\right)}{2} \iint_{\Sigma\left(x^{0}\right)}\left|\frac{\partial \psi}{\partial n}\right|^{2} d \sigma d t
$$

In what follows, we will estimate the first two terms in the left-hand side of (34). First, we have

$$
\left|A_{2}+\frac{N-1}{2} A_{1}\right| \leq 2\left\|\int_{\Omega} \partial_{t} \psi \cdot\left(m_{k} \frac{\partial \psi}{\partial x_{k}}+\frac{N-1}{2} \psi\right)\right\|_{L^{\infty}(0, T)} .
$$

Copyright (c) by SIAM. Unauthorized reproduction of this article is prohibited. 
Second,

$$
\begin{aligned}
& 2\left|\int_{\Omega} \partial_{t} \psi \cdot\left(m_{k} \frac{\partial \psi}{\partial x_{k}}+\frac{N-1}{2} \psi\right)\right| \\
& \leq R\left(x^{0}\right) \int_{\Omega}\left|\partial_{t} \psi\right|^{2}+\frac{1}{R\left(x^{0}\right)} \int_{\Omega}\left|m_{k} \frac{\partial \psi}{\partial x_{k}}+\frac{N-1}{2} \psi\right|^{2} d x \\
& \leq R\left(x^{0}\right) \int_{\Omega}\left(\left|\partial_{t} \psi\right|^{2}+|\nabla \psi|^{2}\right) \\
&-\frac{N(N-1)}{2 R\left(x^{0}\right)} \int_{\Omega}|\psi|^{2}+\frac{(N-1)^{2}}{4 R\left(x^{0}\right)} \int_{\Omega}|\psi|^{2} \\
& \leq R\left(x^{0}\right) \int_{\Omega}\left(\left|\partial_{t} \psi\right|^{2}+|\nabla \psi|^{2}\right)-\frac{N^{2}-1}{4 R\left(x^{0}\right)} \int_{\Omega}|\psi|^{2} \\
& \leq R\left(x^{0}\right) \int_{\Omega}\left(\left|\partial_{t} \psi\right|^{2}+|\nabla \psi|^{2}\right)
\end{aligned}
$$

for all $t \in[0, T]$.

On the other hand, using (33) and taking into account the assumption (15), we find that for all $t$,

$$
\begin{aligned}
E_{0} & =\frac{1}{2} \int_{\Omega}\left(\left|\partial_{t} \psi\right|^{2}+b|\nabla \psi|^{2}-\frac{a^{2}}{4}|\psi|^{2}\right) \\
& \geq \frac{1}{2} \int_{\Omega}\left|\partial_{t} \psi\right|^{2}+\frac{1}{2}\left(b-\frac{a^{2}}{4 \lambda_{1}}\right) \int_{\Omega}|\nabla \psi|^{2} \\
& \geq \frac{1}{2} \min \left(1, b-\frac{a^{2}}{4 \lambda_{1}}\right) \int_{\Omega}\left(\left|\partial_{t} \psi\right|^{2}+|\nabla \psi|^{2}\right),
\end{aligned}
$$

where $\lambda_{1}$ is the first eigenvalue of the Dirichlet Laplacian in $\Omega$. Therefore,

$$
\begin{aligned}
\left|A_{2}+\frac{N-1}{2} A_{1}\right| & \leq R\left(x^{0}\right)\left\|\int_{\Omega}\left(\left|\partial_{t} \psi\right|^{2}+|\nabla \psi|^{2}\right)\right\|_{L^{\infty}(0, T)} \\
& \leq \frac{2 R\left(x^{0}\right)}{\min \left(1, b-\frac{a^{2}}{4 \lambda_{1}}\right)} E_{0}
\end{aligned}
$$

We find from (34) and (35) that

$$
\left(T-\frac{2 R\left(x^{0}\right)}{\min \left(1, b-\frac{a^{2}}{4 \lambda_{1}}\right)}\right) E_{0} \leq \frac{b R\left(x^{0}\right)}{2} \iint_{\Sigma\left(x^{0}\right)}\left|\frac{\partial \psi}{\partial n}\right|^{2} d \sigma d t
$$

Since

$$
T>\tilde{T}_{2}=\frac{2 R\left(x^{0}\right)}{\min \left(1, b-\frac{a^{2}}{4 \lambda_{1}}\right)}=\frac{T\left(x^{0}\right)}{\min \left(1, b-\frac{a^{2}}{4 \lambda_{1}}\right)}
$$

we deduce (27) with

$$
C=\frac{b R\left(x^{0}\right)}{T \min \left(1, b-\frac{a^{2}}{4 \lambda_{1}}\right)-2 R\left(x^{0}\right)} .
$$

This completes the proof.

Copyright $@$ by SIAM. Unauthorized reproduction of this article is prohibited. 
Note. Let us assume that $T>\tilde{T}_{2}$ and let us set

$$
\left\|\left(\varphi^{0}, \varphi^{1}\right)\right\|_{G}^{2}=\iint_{\Sigma\left(x^{0}\right)}\left|\frac{\partial \varphi}{\partial n}\right|^{2} d \sigma d t
$$

for any $\left(\varphi^{0}, \varphi^{1}\right) \in D(A) \times V$, where $\varphi$ is, together with $p$, the solution to (24). Then $\|\cdot\|_{G}$ is a norm in $D(A) \times V$. Let us denote by $G$ the completion of $D(A) \times V$ for this norm. Then $G$ is a Hilbert space and we have

$$
D(A) \times V \hookrightarrow G \hookrightarrow V \times H
$$

with dense and continuous embeddings.

We will now prove a distributed inverse inequality for the solutions to (24).

Lemma 3.2. Let $\mathcal{O}, a$, and $b$ satisfy the assumptions in Theorem 1.3. There exists $T_{2}=T_{2}\left(a, b, \Omega, x^{0}, \delta\right)$ with the following property: for any $T>T_{2}$, there exists a positive constant $C$ only depending on $a, b, \Omega, x^{0}, \delta$, and $T$ such that

$$
\left\|\left(\varphi^{0}, \varphi^{1}\right)\right\|_{H \times V^{\prime}}^{2} \leq C \iint_{\mathcal{O} \times(0, T)}|\varphi|^{2}
$$

for any $\left(\varphi^{0}, \varphi^{1}\right) \in H \times V^{\prime}$, where $\varphi$ is, together with some $p$, the associated solution to (24).

Proof. Let us again introduce the change of variables (28). It is then clear that it suffices to prove that there exists $T_{2}$ such that for all $T>T_{2}$, one has

$$
\left\|\left(\psi^{0}, \psi^{1}\right)\right\|_{H \times V^{\prime}}^{2} \leq C \iint_{\mathcal{O} \times(0, T)}|\psi|^{2}
$$

for all $\left(\psi^{0}, \psi^{1}\right) \in H \times V^{\prime}$, where $C$ only depends on $a, b, \Omega, x^{0}, \delta$, and $T$.

Let $\gamma$ and $\tilde{T}_{2}$ be given by

$$
\gamma=\bigcup_{x \in \Gamma\left(x^{0}\right)} B(x ; \delta / 2) \cap \partial \Omega, \quad \tilde{T}_{2}=\frac{T\left(x^{0}\right)}{\min \left(1, b-a^{2} /\left(4 \lambda_{1}\right)\right)} .
$$

Let us assume that $T>\tilde{T}_{2}$ and let $\alpha>0$ be such that $T-2 \alpha>\tilde{T}_{2}$. We will assume for the moment that there exists $C(\delta)>0$ such that for all $\left(u^{0}, u^{1}\right) \in V \times H$, the solution $u$ to $(29)$ with final data $\left(u^{0}, u^{1}\right)$ satisfies

$$
\left\|\left(u^{0}, u^{1}\right)\right\|_{V \times H}^{2} \leq C(\delta) \iint_{\mathcal{G}_{\delta}\left(x^{0}\right) \times(0, T)}\left|\partial_{t} u\right|^{2} .
$$

Let $\left(\psi^{0}, \psi^{1}\right) \in H \times V^{\prime}$ be given, let us set $u^{1}=\psi^{0}$, let $u^{0}$ be the unique function satisfying

$$
\left(\left(u^{0}, w\right)\right)=\left\langle\psi^{1}, w\right\rangle \quad \forall w \in V, u^{0} \in V
$$

(i.e., $u^{0}=A_{0}^{-1} \psi^{1}$ ), and let us introduce $u$ with

$$
u(t)=u^{0}+\int_{t}^{T} \psi(s) d s \quad \forall t \in[0, T]
$$

Copyright $@$ by SIAM. Unauthorized reproduction of this article is prohibited. 
where $\psi$ is (together with some $q$ ) the solution to (29). It is then easy to check that $u$ is, together with some pressure $h$, the unique solution to (29)with final data $\left(u^{0}, u^{1}\right)$. Moreover,

$$
\left\|\left(u^{0}, u^{1}\right)\right\|_{V \times H}^{2}=\left\|\left(\psi^{0}, \psi^{1}\right)\right\|_{H \times V^{\prime}}^{2} .
$$

Consequently, we see from (37) that

$$
\left\|\left(\psi^{0}, \psi^{1}\right)\right\|_{H \times V^{\prime}}^{2} \leq C(\delta) \iint_{\mathcal{G}_{\delta}\left(x^{0}\right) \times(0, T)}|\psi|^{2}
$$

for all $\left(\psi^{0}, \psi^{1}\right) \in H \times V^{\prime}$.

This shows that in order to get (36), we only have to prove (37).

Let us now establish (37). Obviously, it will suffice to prove this inequality when $\left(u^{0}, u^{1}\right) \in D(A) \times V$. To this aim, we will use Lemma 3.1 and, more precisely, that if $T>\tilde{T}_{2}$, there exists $C>0$ such that

$$
\left\|\left(u^{0}, u^{1}\right)\right\|_{V \times H}^{2} \leq C \iint_{\gamma \times(0, T)}\left|\frac{\partial u}{\partial n}\right|^{2} d \sigma d t
$$

for every $\left(u^{0}, u^{1}\right) \in D(A) \times V$.

Observe that if $\varepsilon>0$ is such that $T-2 \varepsilon>\tilde{T}_{2}$, we still have

$$
\left\|\left(u^{0}, u^{1}\right)\right\|_{V \times H}^{2} \leq C \iint_{\gamma \times(\varepsilon, T-\varepsilon)}\left|\frac{\partial u}{\partial n}\right|^{2} d \sigma d t
$$

for some $C=C\left(a, b, \Omega, x^{0}, T\right)$.

Now, let $\varepsilon^{\prime}, \delta^{\prime}$, and $\delta^{\prime \prime}$ be such that

$$
\delta / 2<\delta^{\prime}<\delta^{\prime \prime}<\delta \text { and } 0<\varepsilon^{\prime}<\varepsilon .
$$

Let $\ell=\ell(x, t)$ be a vector field in $W^{1, \infty}(Q)^{N}$ such that

$$
\begin{aligned}
& \ell(x, t)=\vec{n}(x) \quad \forall(x, t) \in \gamma \times(\varepsilon, T-\varepsilon), \\
& \ell(x, t)=0 \quad \forall(x, t) \in \bar{Q} \quad \text { with } t \leq \varepsilon^{\prime} \text { or } t \geq T-\varepsilon^{\prime}, \\
& \ell(x, t)=0 \quad \forall(x, t) \in \bar{Q} \quad \text { with } x \notin \mathcal{G}_{\delta^{\prime}}\left(x^{0}\right), \\
& \ell(x, t) \cdot \vec{n}(x) \geq 0 \quad \forall(x, t) \in \gamma \times(0, T) .
\end{aligned}
$$

Multiplying the equation satisfied by $u$ and $h$ by $\ell \cdot \nabla u$ and integrating in $Q$, we easily obtain

$$
\begin{aligned}
\iint_{\gamma \times(\varepsilon, T-\varepsilon)}\left|\frac{\partial u}{\partial n}\right|^{2} d \sigma d t & \leq \iint_{\partial \Omega \times\left(\varepsilon^{\prime}, T-\varepsilon^{\prime}\right)} \ell \cdot \vec{n}\left|\frac{\partial u}{\partial n}\right|^{2} d \sigma d t \\
& \leq C \iint_{\mathcal{G}_{\delta^{\prime}}\left(x^{0}\right) \times\left(\varepsilon^{\prime}, T-\varepsilon^{\prime}\right)}\left(|u|^{2}+\left|\partial_{t} u\right|^{2}+|\nabla u|^{2}+|h||\nabla u|\right) .
\end{aligned}
$$

For any small $\kappa>0$, we have that

$$
\begin{aligned}
\iint_{\mathcal{G}_{\delta^{\prime}}\left(x^{0}\right) \times\left(\varepsilon^{\prime}, T-\varepsilon^{\prime}\right)}|h||\nabla u| & \leq \kappa\|h\|_{L^{2}(Q)}^{2}+C(\kappa) \iint_{\mathcal{G}_{\delta^{\prime}}\left(x^{0}\right) \times\left(\varepsilon^{\prime}, T-\varepsilon^{\prime}\right)}|\nabla u|^{2} \\
& \leq C \kappa\left\|\left(u^{0}, u^{1}\right)\right\|_{V \times H}^{2}+C(\kappa) \iint_{\mathcal{G}_{\delta^{\prime}}\left(x^{0}\right) \times\left(\varepsilon^{\prime}, T-\varepsilon^{\prime}\right)}|\nabla u|^{2},
\end{aligned}
$$

Copyright $@$ by SIAM. Unauthorized reproduction of this article is prohibited. 
in view of Theorem 2.1. On the other hand, using localized energy estimates, we find easily that

$$
\begin{aligned}
\iint_{\mathcal{G}_{\delta^{\prime}}\left(x^{0}\right) \times\left(\varepsilon^{\prime}, T-\varepsilon^{\prime}\right)}|\nabla u|^{2} & \leq C \iint_{\mathcal{G}_{\delta^{\prime \prime}}\left(x^{0}\right) \times(0, T)}\left(|u|^{2}+\left|\partial_{t} u\right|^{2}+|h||u|\right) \\
& \leq \kappa\|h\|_{L^{2}(Q)}^{2}+C(\kappa) \iint_{\mathcal{G}_{\delta^{\prime \prime}}\left(x^{0}\right) \times(0, T)}\left(|u|^{2}+\left|\partial_{t} u\right|^{2}\right) \\
& \leq C \kappa\left\|\left(u^{0}, u^{1}\right)\right\|_{V \times H}^{2}+C(\kappa) \iint_{\mathcal{G}_{\delta^{\prime \prime}}\left(x^{0}\right) \times(0, T)}\left(|u|^{2}+\left|\partial_{t} u\right|^{2}\right) .
\end{aligned}
$$

Consequently, we also have the following estimate for any small $\kappa>0$ :

$$
\begin{aligned}
& \iint_{\gamma \times(\varepsilon, T-\varepsilon)}\left|\frac{\partial u}{\partial n}\right|^{2} d \sigma d t \\
& \quad \leq C \kappa\left\|\left(u^{0}, u^{1}\right)\right\|_{V \times H}^{2}+C(\kappa) \iint_{\mathcal{G}_{\delta}\left(x^{0}\right) \times(0, T)}\left(|u|^{2}+\left|\partial_{t} u\right|^{2}\right) .
\end{aligned}
$$

In view of (38), by choosing $\kappa$ small enough, we deduce that

$$
\left\|\left(u^{0}, u^{1}\right)\right\|_{V \times H}^{2} \leq C \iint_{\mathcal{G}_{\delta}\left(x^{0}\right) \times(0, T)}\left(|u|^{2}+\left|\partial_{t} u\right|^{2}\right) .
$$

Finally, using a straighforward uniqueness-compactness argument (see, for example, Appendix 1 in [15] for a similar argument), we can eliminate the zero-order term $|u|^{2}$ in the previous estimate.

This gives (37) for a new constant $C=C(\delta)$ and ends the proof.

3.2. The distributed exact controllability result. The goal of this section is to give the proof of Theorem 1.3. This will be possible thanks to the inverse inequality in Lemma 3.2.

We can easily connect the controllability properties of systems (4) and (9). More precisely, the exact controllability of (9) starting from $\left(0, y_{0}\right)$ is equivalent to the exact controllability for the velocity field $y$ and the stress tensor $\tau$ in (5) starting from $\left(y_{0}, \tau_{0}\right)$.

We have the following result, which provides the exact controllability of (9).

TheOREM 3.3. Let $\mathcal{O}, a, b$, and $\tau_{0}$ satisfy the assumptions of Theorem 1.3 and let us assume that $T>T_{2}$. For any $\left(z^{0}, z^{1}\right),\left(w^{0}, w^{1}\right) \in V \times H$ there exists a control $u \in L^{2}(\mathcal{O} \times(0, T))^{N}$ such that the unique solution to the associated problem (9) satisfies

$$
\left(z(T), \partial_{t} z(T)\right)=\left(w^{0}, w^{1}\right) .
$$

Proof. Let us give the sketch of the proof of Theorem 3.3. We will adapt the HUM of J.-L. Lions $[14,15]$. The argument is very classical and well known; consequently, we only give a sketch of the proof.

Since (9) is linear and reversible in time (up to a change of sign of $a$ ), it can be assumed that $\tau_{0}=0$ and it suffices to prove a null controllability property, that is, (39) for $\left(w^{0}, w^{1}\right)=(0,0)$.

On the other hand, the change of variables

$$
z^{*}=e^{-a t / 2} z, \quad Z^{*}=e^{-a t / 2} Z
$$

Copyright $\odot$ by SIAM. Unauthorized reproduction of this article is prohibited. 
allows us to rewrite (9) (with $\tau_{0}=0$ ) as follows:

$$
\begin{cases}z_{t t}^{*}-b \Delta z^{*}-\frac{a^{2}}{4} z^{*}+\nabla Z^{*}=u 1_{\mathcal{O}} & \text { in } Q \\ \nabla \cdot z^{*}=0 & \text { in } Q \\ z^{*}=0 & \text { on } \Sigma \\ z^{*}(0)=z^{0}, \quad \partial_{t} z^{*}(0)=z^{1}-\frac{a}{2} z^{0} & \text { in } \Omega\end{cases}
$$

and it is obviously sufficient to prove that the same property holds for (40).

Let us assume that $\left(\varphi^{0}, \varphi^{1}\right) \in H \times V^{\prime}$ and let us consider the following homogeneous problem:

$$
\begin{cases}\partial_{t t} \varphi-b \Delta \varphi-\frac{a^{2}}{4} \varphi+\nabla p=0 & \text { in } Q \\ \nabla \cdot \varphi=0 & \text { in } Q \\ \varphi=0 & \text { on } \Sigma \\ \varphi(0)=\varphi^{0}, \quad \partial_{t} \varphi(0)=\varphi^{1} & \text { in } \Omega .\end{cases}
$$

This problem admits a unique solution $(\varphi, p)$ with

$$
\varphi \in C^{0}([0, T] ; H), \quad \partial_{t} \varphi \in C^{0}\left([0, T] ; V^{\prime}\right) .
$$

Let us now introduce the following backward problem:

$$
\begin{cases}\partial_{t t} \psi-b \Delta \psi-\frac{a^{2}}{4} \psi+\nabla q=\varphi 1_{\mathcal{O}} & \text { in } Q, \\ \nabla \cdot \psi=0 & \text { in } Q, \\ \psi=0 & \text { on } \Sigma, \\ \psi(T)=0, \quad \partial_{t} \psi(T)=0 & \text { in } \Omega .\end{cases}
$$

Again, this problem possesses exactly one solution $\psi$. Now,

$$
\psi \in C^{0}([0, T] ; V), \quad \partial_{t} \psi \in C^{0}([0, T] ; H) .
$$

Let us introduce the linear operator $\mathcal{B}: H \times V^{\prime} \mapsto H \times V$ with

$$
\mathcal{B}\left(\varphi^{0}, \varphi^{1}\right)=\left(\partial_{t} \psi(0),-\psi(0)\right) \quad \forall\left(\varphi^{0}, \varphi^{1}\right) \in H \times V^{\prime} .
$$

Then $\mathcal{B}$ is a well-defined linear continuous mapping.

Let us consider an initial data $\left(\xi^{0}, \xi^{1}\right) \in H \times V^{\prime}$ and let us denote by $(\xi, \Xi)$ the solution to the corresponding problem (41). Then, taking into account the definition of $\mathcal{B}$, it is clear that

$$
\left\langle\mathcal{B}\left(\varphi^{0}, \varphi^{1}\right),\left(\xi^{0}, \xi^{1}\right)\right\rangle=\iint_{\mathcal{O} \times(0, T)} \varphi \cdot \xi
$$

for all $\left(\varphi^{0}, \varphi^{1}\right) \in H \times V^{\prime}$, where $\langle\cdot, \cdot\rangle$ denotes the duality pairing associated to $H \times V^{\prime}$ and $H \times V$. In particular, we have

$$
\left\langle\mathcal{B}\left(\varphi^{0}, \varphi^{1}\right),\left(\varphi^{0}, \varphi^{1}\right)\right\rangle=\iint_{\mathcal{O} \times(0, T)}|\varphi|^{2}:=\left\|\left(\varphi^{0}, \varphi^{1}\right)\right\|_{F}^{2}
$$

for all $\left(\varphi^{0}, \varphi^{1}\right) \in V \times H$. 
Since $T>T_{2}$, we know from Lemma 3.2 that

$$
\left\|\left(\varphi^{0}, \varphi^{1}\right)\right\|_{F}:=\left(\iint_{\mathcal{O} \times(0, T)}|\varphi|^{2}\right)^{1 / 2}
$$

is a norm in $H \times V^{\prime}$ equivalent to the usual product norm. Consequently, for any $\left(z^{0}, z^{1}\right) \in V \times H$ there exists a unique $\left(\varphi^{0}, \varphi^{1}\right) \in H \times V^{\prime}$ satisfying

$$
\mathcal{B}\left(\varphi^{0}, \varphi^{1}\right)=\left(z^{1}-\frac{a}{2} z^{0}, z^{0}\right) .
$$

Let us take in (40) the control $u=\varphi 1_{\mathcal{O}}$, where $\varphi$ is, together with $p$, the solution to (41) associated to this $\left(\varphi^{0}, \varphi^{1}\right)$. Then, taking into account the definition of $\mathcal{B}$, it is obvious that

$$
\left(z^{*}(T), \partial_{t} z^{*}(T)\right)=(0,0) .
$$

Thus, we have solved the null controllability problem for (40) with a control $u \in$ $L^{2}(\mathcal{O} \times(0, T))^{N}$ and an associated state that starts from the initial data $\left(z^{0}, z^{1}-\frac{a}{2} z^{0}\right)$.

This ends the proof.

We can now achieve the proof of Theorem 1.3.

Let the assumptions in this theorem be satisfied and let $T, y_{0}, y_{d}, \tau_{0}$, and $\tau_{d}$ be given with $T>T_{2}, y_{0}, y_{d} \in H, \tau_{0} \in \Phi$, and $\tau_{d} \in \Phi^{*}\left(\tau_{0}\right)$. Let us show that there exists $v \in L^{2}(\mathcal{O} \times(0, T))^{N}$ such that the solution to (4) satisfies (14).

Let us set

$$
z^{0}=0, \quad z^{1}=y_{0}, \quad w^{1}=e^{a T} y_{d}
$$

and let us choose $w^{0} \in V$ such that

$$
\tau_{d}=e^{-a T} \tau_{0}+b D w^{0} .
$$

Then, using again (8), it becomes clear that what we have to prove is that for some $u \in L^{2}(\mathcal{O} \times(0, T))^{N}$, the solution to (9) satisfies (39). But this is true in view of Theorem 3.3.

Consequently, Theorem 1.3 holds.

3.3. The boundary exact controllability result. This section is devoted to proving Theorem 1.4. Thus, let us consider the system (5), where $T>\tilde{T}_{2}$ and $\left(y_{0}, \tau_{0}\right) \in \tilde{H} \times \Phi$, and let us fix $y_{d} \in H$ and $\tau_{d} \in \Phi^{*}\left(\tau_{0}\right)$.

Our aim is to find a control $r \in L^{2}(\gamma \times(0, T))^{N}$ and an associated solution to (5) satisfying (14).

Since $\gamma$ is an open neighborhood of $\Gamma\left(x^{0}\right)$ in $\partial \Omega$, there exists a bounded regular open set $D \supset \Omega$ with the following two properties:

- If $z \in H_{0}^{1}(D)$, then $z=0$ on $\partial \Omega \backslash \gamma$.

- Let us set

$$
\Lambda\left(x^{0}\right)=\left\{x \in \partial D:\left(x-x^{0}\right) \cdot \vec{n}(x)>0\right\} .
$$

Then, for some $\delta>0$, one has

$$
\mathcal{H}_{\delta}\left(x^{0}\right):=\bigcup_{x \in \Lambda\left(x^{0}\right)} B(x ; \delta) \cap D \subset D \backslash \bar{\Omega} .
$$

- Let us denote by $\tilde{S}_{2}$ the time $\tilde{T}_{2}$ relative to $D$. Then $T>\tilde{S}_{2}$. 
As in section 2.2, we can denote by $H(D), V(D)$, and $\Phi(D)$ the spaces $H, V$, and $\Phi$ relative to $D$ and we can extend by zero the functions $y_{0}, y_{d}, \tau_{0}$, and $\tau_{d}$ to get $\hat{y}_{o}, \hat{y}_{d} \in H(D), \hat{\tau}_{0} \in \Phi(D)$, and $\hat{\tau}_{d} \in \Phi(D)$ with $\hat{\tau}_{d}=e^{-a T} \hat{\tau}_{0}+D \hat{w}$ for some $\hat{w} \in V(D)$.

In view of Theorem 3.3, there exist $L^{2}$ controls such that the solution to the auxiliary problem (26) satisfies

$$
\hat{y}(T)=\hat{y}_{d}, \quad \hat{\tau}(T)=\hat{\tau}_{d} .
$$

Then, the trace on $\gamma \times(0, T)$ of $\hat{y}$ and the restrictions to $Q$ of the functions $\hat{y}, \hat{\pi}$, and $\hat{\tau}$ solve the considered exact controllability problem.

This proves Theorem 1.4.

\section{Further remarks and open questions.}

4.1. Exact controllability of linear Maxwell fluids with general controls. The exact controllability of the Maxwell models (4) and (5) with controls supported by more general sets is unknown. To our knowledge, we only have "abstract" results. Thus, for instance, the following holds:

Assume that, for instance, $\tau_{0}=0$ and $T>T_{1}$ (this is the minimal time furnished by Theorem 1.1). Then there exists a Hilbert space $F_{1}$ that contains $\mathcal{V}$ as a dense subspace such that for any $y_{0} \in F_{1}^{\prime} \cap H$ there exist controls $v \in L^{2}(\mathcal{O} \times(0, T))^{N}$ such that the corresponding solutions to (4) satisfy

$$
y(T)=0, \quad \tau(T)=0 .
$$

The proof can be obtained by adapting again the HUM method of [15]. For completeness, let us present a sketch.

First, by means of the change of variables (8), we see again that (4) is equivalent to (9) with $\left(z^{0}, z^{1}\right)=\left(0, y_{0}\right)$ and the null controllability of $(9)$ starting from $\left(0, y_{0}\right)$ is equivalent to the null controllability of (4) starting from $\left(y_{0}, \tau_{0}\right)$.

On the other hand, it can be proved that whenever $y^{0}$ is chosen in an appropriate space, (9) is null controllable if $T>T_{1}$ and $\tau_{0}=0$. More precisely, let $F_{0}$ be the completion of $\mathcal{V} \times \mathcal{V}$ for the norm

$$
\left\|\left(\varphi^{0}, \varphi^{1}\right)\right\|_{F_{0}}=\|\varphi\|_{L^{2}(\mathcal{O} \times(0, T))},
$$

where $\varphi$ is the solution to the adjoint system (24). Notice that $\|\cdot\|_{F_{0}}$ is a true norm in $\mathcal{V} \times \mathcal{V}$ (because $T>T_{1}$ and, consequently, the previous adjoint system satisfies the unique continuation property). Let $F_{0}^{\prime}$ be the dual space of $F_{0}$. Then, for any $\left(z^{0}, z^{1}\right)$ such that $\left(z^{1}-a z^{0},-z^{0}\right) \in F_{0}^{\prime}$, there exist controls $u \in L^{2}(\mathcal{O} \times(0, T))^{N}$ such that the corresponding solution of (9) satisfies

$$
z(T)=0, \quad \partial_{t} z(T)=0 .
$$

In particular, setting $F_{0}=F_{1} \times F_{2}$ and $F_{0}^{\prime}=F_{1}^{\prime} \times F_{2}^{\prime}$, we see that whenever $T>T_{1}$ and $\tau_{0}=0$, for each $y_{0} \in F_{1}^{\prime}$ there exists a control $v \in L^{2}(\mathcal{O} \times(0, T))^{N}$ such that the solution to (4) satisfies (43).

This proves our assertion.

The space $F_{0}$ is defined as the completion of $\mathcal{V} \times \mathcal{V}$ for a suitable norm and, obviously, depends on $a, b, \Omega, \mathcal{O}$, and $T$. Unfortunately, it is not easy to identify $F_{0}$ 
in terms of usual functional spaces (and this is why we say that we are only able to obtain "abstract" results).

Mainly, this is because we do not know how to prove suitable inverse inequalities. For instance, if $\Psi=\Psi_{1} \times \Psi_{2}$ were an easy-to-handle Banach space containing $\mathcal{V} \times \mathcal{V}$ as a dense subspace and the inverse inequality

$$
\left\|\left(\varphi^{0}, \varphi^{1}\right)\right\|_{\Psi}^{2} \leq C \iint_{\mathcal{O} \times(0, T)}|\varphi|^{2} \quad \forall\left(\varphi^{0}, \varphi^{1}\right) \in \mathcal{V} \times \mathcal{V}
$$

were satisfied, then we would have $F_{0} \subset \Psi$ with a continuous and dense embedding and, therefore, we would also have $F^{\prime} \supset \Psi^{\prime}$. The consequence would be that any initial $\left(y_{0}, 0\right) \in \Psi^{\prime}$ can be driven exactly to zero with controls $v \in L^{2}(\mathcal{O} \times(0, T))^{N}$ at time $T$.

A related question is the following: which conditions on $\mathcal{O}$ and $T$ ensure that (44) holds, for instance, for $\Psi=H \times V^{\prime}$ ?

In view of what is known for the wave equation, it seems reasonable to expect that the well-known geometric control conditions on $\mathcal{O}$ and $T$ suffice. In fact, it is maybe possible to adapt the microlocal analysis techniques in [1] and/or the Carleman techniques in $[18,27]$ to this context. But, at present, this is an open question.

4.2. On the exact controllability of linear Maxwell fluids for large $a^{2} / b$. For large $a^{2} / b$, the arguments in section 3 cannot be applied and do not lead to exact controllability directly. What we need is, again, an inverse inequality of the form

$$
\left\|\left(\psi^{0}, \psi^{1}\right)\right\|_{V \times H}^{2} \leq C \iint_{\gamma \times(0, T)}\left|\frac{\partial \psi}{\partial n}\right|^{2} d \sigma d t
$$

for the solutions to $(29)$ with $\left(\psi^{0}, \psi^{1}\right) \in D(A) \times V$.

A natural and quite tempting strategy to get (45) is to apply a compactnessuniqueness argument. Thus, we write the solution to (29) in the form $\psi=\eta+\zeta$, where $\eta$ is, together with some $\beta$, the solution to

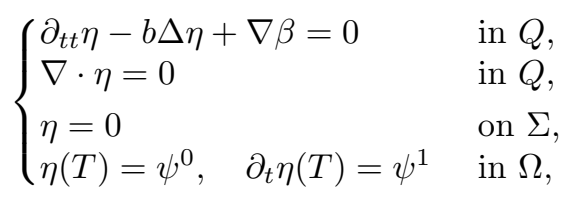

and $\zeta$ is, together with some pressure, the solution to $(29)$ for $\left(\psi^{0}, \psi^{1}\right) \equiv(0,0)$ with the right-hand side $f \equiv a^{2} \eta / 4$.

Assume that $T>\tilde{T}_{2}$. Then, in view of Lemma 3.1, one has

$$
\begin{aligned}
\left\|\left(\psi^{0}, \psi^{1}\right)\right\|_{V \times H}^{2} & \leq C \iint_{\gamma \times(0, T)}\left|\frac{\partial \eta}{\partial n}\right|^{2} d \sigma d t \\
& \leq C \iint_{\gamma \times(0, T)}\left|\frac{\partial \psi}{\partial n}\right|^{2} d \sigma d t+C \iint_{\gamma \times(0, T)}\left|\frac{\partial \zeta}{\partial n}\right|^{2} d \sigma d t \\
& \equiv C \iint_{\gamma \times(0, T)}\left|\frac{\partial \psi}{\partial n}\right|^{2} d \sigma d t+C\left\|\tilde{\Lambda}\left(\psi^{0}, \psi^{1}\right)\right\|_{L^{2}(\gamma \times(0, T)}^{2} .
\end{aligned}
$$

Consequently, in order to deduce (45), it would be sufficient to check that

- $\tilde{\Lambda}: V \times H \mapsto L^{2}(\Sigma)^{N}$ is well defined, linear, and compact and

- $\left.\left(\psi^{0}, \psi^{1}\right) \mapsto \frac{\partial \psi}{\partial n}\right|_{\gamma \times(0, T)}$ is one-to-one. 
The first of these assertions is relatively easy to prove. However, the second one (a unique continuation property) is an open question. As in the previous section, it is reasonable to expect that appropriate techniques lead to a positive answer, at least for $T$ sufficiently large, depending on $a$. But this is unknown.

4.3. Controllability problems for nonlinear Maxwell system. In control theory, it is relatively frequent to deduce local controllability properties for a nonlinear state equation from the controllability of its linearization at a well-chosen state.

Thus, for instance, it should make sense to view (4) as the linearization at zero of (6) and to try to use Theorem 1.3 as a first step in the proof of the following local exact controllability property: there exists $\kappa>0$ such that whenever $y_{0}, y_{d} \in H$, $\tau_{0} \in \Phi, \tau_{d} \in \Phi$, and

$$
\left\|y_{0}\right\|+\left\|y_{d}\right\|+\left\|\tau_{0}\right\|+\left\|\tau_{d}\right\| \leq \kappa,
$$

there exists $v \in L^{2}(\mathcal{O} \times(0, T))^{N}$ such that the associated solution satisfies (14).

However, there are reasons to think that this will not be easy. First, several recent works by Renardy show that the solutions to (4) necessarily satisfy, even in the case of (simplified) parallel shear flows, particular structural properties of the form

$$
\tau(T) \in \mathcal{R}\left(\tau_{0}, T\right)
$$

where $\mathcal{R}\left(\tau_{0}, T\right)$ is a nontrivial subset of $\Phi$ that can be complicated and not easy to identify; see $[20,21,23,25]$. Furthermore, even if we were able to characterize attainable states, we would have to find an appropriate mathematical framework to formulate the problem and pass from the linear to the nonlinear setting (in short, an adequate inverse function theorem).

Appendix A. A technical result. In this section, we will give a sketch of the proof of Theorem 2.1.

Proof. Let us present the sketch of the proof of Theorem 2.1. This result is implied by classical semigroup theory; see, for example, [5, 17, 26]. For instance, let us prove the results that have been claimed when $f=0$ and $\left(z^{0}, z^{1}\right) \in V \times H$.

We can introduce the Hilbert space $X=V \times H$ and the unbounded operator $B: D(B) \mapsto X$ with $D(B)=D(A) \times V$ and

$$
B(z, w)=(w+a z, b A z+c z) \quad \forall(z, w) \in D(B),
$$

where $A: D(A) \mapsto H$ is the usual Stokes operator.

It is not difficult to check that $B$ is the generator of a continuous group $\{S(t)\}_{t \in \mathbb{R}}$ of bounded linear operators in $X$. Consequently, for all $\left(z^{0}, z^{1}\right) \in V \times H$, the function $t \mapsto S(t)\left(z^{0}, z^{1}-a z^{0}\right)$ is well defined and continuous and provides a solution to the Cauchy problem

$$
\left\{\begin{array}{l}
\frac{d z}{d t}=w+a z, \quad \frac{d w}{d t}=b A z+c z, \quad t \in \mathbb{R} \\
z(0)=z^{0}, \quad w(0)=z^{1}-a z^{0}
\end{array}\right.
$$

Taking into account the definition of the Stokes operator and using the classical De Rham lemma, we find that $z$ is, together with some $Z$, the unique solution to (16) with $f=0$ and satisfies the required properties. 
The regularity and estimate of $Z$ can be established as follows. Let us first assume that $\left(z^{0}, z^{1}\right) \in D(A) \times V$ and let us normalize $Z$ by imposing

$$
\int_{\Omega} Z=0 \text { in }(0, T) .
$$

Then, for some constants $C=C(a, b, c, \Omega, T)$, we have

$$
\begin{aligned}
|Z(t)|^{2} & \leq C\|\nabla Z(t)\|_{H^{-1}}^{2} \\
& \leq C\left(\left\|\partial_{t t} z(t)\right\|_{H^{-1}}^{2}+\left\|\partial_{t} z(t)\right\|_{H^{-1}}^{2}+\|z(t)\|_{H^{-1}}^{2}+\|\Delta z(t)\|_{H^{-1}}^{2}\right) \\
& \leq C\left\|\partial_{t t} z(t)\right\|_{H^{-1}}^{2}+C\left\|\left(z^{0}, z^{1}\right)\right\|_{V \times H}^{2} .
\end{aligned}
$$

Also,

$$
\begin{aligned}
\int_{0}^{T}\left\|\partial_{t t} z(t)\right\|_{H^{-1}}^{2} d t= & \int_{0}^{T}\left\langle\partial_{t t} z(t),(-\Delta)^{-1} \partial_{t t} z(t)\right\rangle_{H^{-1}, H_{0}^{1}} d t \\
= & \int_{0}^{T}\left\langle a \partial_{t} z(t)+b \Delta z(t)-c z(t)-\nabla Z(t),(-\Delta)^{-1} \partial_{t t} z(t)\right\rangle_{H^{-1}, H_{0}^{1}} d t \\
= & -b \int_{0}^{T}\left(z(t), \partial_{t t} z(t)\right) d t \\
& +\int_{0}^{T}\left\langle a \partial_{t} z(t)-c z(t)-\nabla Z(t),(-\Delta)^{-1} \partial_{t t} z(t)\right\rangle_{H^{-1}, H_{0}^{1}} d t \\
\leq & -b \int_{0}^{T}\left(z(t), \partial_{t t} z(t)\right) d t+C\left\|\left(z^{0}, z^{1}\right)\right\|_{V \times H}^{2}
\end{aligned}
$$

and, finally,

$$
-\int_{0}^{T}\left(z(t), \partial_{t t} z(t)\right) d t=\int_{0}^{T}\left|\partial_{t} z(t)\right|^{2} d t-\left.\left(z, \partial_{t} z\right)\right|_{t=0} ^{t=T} \leq C\left\|\left(z^{0}, z^{1}\right)\right\|_{V \times H}^{2}
$$

These estimates show that $Z \in L^{2}\left(0, T ; L^{2}(\Omega)\right)$ and

$$
\|Z\|_{L^{2}\left(0, T ; L^{2}\right)} \leq C\left\|\left(z^{0}, z^{1}\right)\right\|_{V \times H}
$$

for some $C=C(a, b, c, \Omega, T)$. Now, an elementary density argument allows us to deduce the same for general data $\left(z^{0}, z^{1}\right) \in V \times H$.

\section{REFERENCES}

[1] C. Bardos, G. Lebeau, And J. RAuch, Sharp sufficient conditions for the observation, control, and stabilization of waves from the boundary, SIAM J. Control Optim., 30 (1992), pp. 10241065.

[2] L. Bers, F. John, And M. Schechter, Partial Differential Equations, John Wiley \& Sons, New York, 1964.

[3] J.-M. Coron, On the controllability of the 2-D incompressible Navier-Stokes equations with the Navier slip boundary conditions, ESAIM Control Optim. Calc. Var., 1 (1996), pp. 35-75.

[4] J.-M. Coron And A.V. Fursikov, Global exact controllability of the $2 D$ Navier-Stokes equations on a manifold without boundary, Russ. J. Math. Phys., 4 (1996), pp. 429-448.

[5] R. Dautray and J.L. Lions, Mathematical Analysis and Numerical Methods for Science and Technology, Vol. 6: Evolution Problems, Springer-Verlag, Berlin, 1993.

[6] C. FABRE, Uniqueness results for Stokes equations and their consequences in linear and nonlinear control problems, ESAIM Control Optim. Calc. Var., 1 (1996), pp. 267-302.

Copyright $@$ by SIAM. Unauthorized reproduction of this article is prohibited. 
[7] C. Fabre and G. Lebeau, Prolongement unique des solutions des équations de Stokes, Comm. Partial Differential Equations, 21 (1996), pp. 573-596.

[8] E. Fernández-Cara, S. Guerrero, O.Yu. Imanuvilov, and J.-P. Puel, Local exact controllability of the Navier Stokes system, J. Math. Pures Appl., 83 (2004), pp. 1501-1542.

[9] E. Fernández-Cara, S. Guerrero, O.Yu. Imanuvilov, and J.-P. Puel, Some controllability results for the $N$-dimensional Navier-Stokes and Boussinesq systems with $N-1$ scalar controls, SIAM J. Control Optim., 45 (2006), pp. 146-173.

[10] A.V. Fursikov, And O.Yu. Imanuvilov, Exact controllability of the Navier-Stokes and Boussinesq equations, Uspekhi Mat. Nauk, 3 (1999), pp. 93-146 (in Russian); Russian Math. Surveys, 3 (1999), pp. 565-618 (in English).

[11] L.F. Ho, Observabilité frontière de l'équation des ondes, C. R. Acad. Sci. Paris Sér. I Math., 302 (1986), pp. 443-446.

[12] O.Yu. ImanuviLOv, On exact controllability for the Navier-Stokes equations, ESAIM Control Optim. Calc. Var., 3 (1998), pp. 97-131.

[13] D.D. Joseph, Fluid Dynamics of Viscoelastic Liquids, Appl. Math. Sci. 84, Springer-Verlag, New York, 1990.

[14] J.-L. LIONS, Exact controllability, stabilization and perturbations for distributed systems, SIAM Rev., 30 (1988), pp. 1-68.

[15] J.-L. Lions, Contrôlabilité exacte perturbation et stabilisation de systèmes distribués, Tomes 1, 2, Contrôlabilité exacte, Masson, Paris, 1988.

[16] A. Osses, A rotated multiplier applied to the controllability of the waves, elasicity and tangencial Stokes control, SIAM J. Control Optim., 40 (2001), pp. 777-800.

[17] A. PAZY, Semigroups of Linear Operators and Applications to Partial Differential Equations, Appl. Math. Sci. 44, Springer-Verlag, New York, 1983.

[18] J.-P. Puel, Application of Carleman inequalities to controllability and inverse problems, Textos Metodos Mat. Instituto de Matematica de l'UFRJ, to appear.

[19] M. Renardy, Are viscoelastic flows under control or out of control?, Systems Control Lett., 54 (2005), pp. 1183-1193.

[20] M. Renardy, Shear flow of viscoelastic fluids as a control problem, J. Non-Newtonian Fluid Mech., 131 (2005), pp. 59-63.

[21] M. Renardy, On control of shear flow of an upper convected Maxwell fluid, Z. Angew. Math. Mech., 87 (2007), pp. 213-218.

[22] M. Renardy, A note on a class of observability problems for PDEs, Systems Control Lett., 58 (2009), pp. 183-187.

[23] M. REnARDY, Controllability of viscoelastic stresses for nonlinear Maxwell models, J. NonNewtonian Fluid Mech., 156 (2009), pp. 70-74.

[24] M. Renardy, W.J. Hrusa, And J.A. Nohel, Mathematical problems in viscoelasticity, Pitman Monogr. Surveys in Pure and Appl. Math. 35, Longman Scientific \& Technical, Essex, UK, 1987.

[25] E. Savelev and M. Renardy, Control of homogeneous shear flow of multimode Maxwell fluids, J. Non-Newtonian Fluid Mech., 165 (2010), pp. 136-142.

[26] K. Yosida, Functional Analysis, Classics Math., Springer-Verlag, Berlin, 1995.

[27] X. Zhang, Explicit observability estimate for the wave equation with potential and its application, R. Soc. Lond. Proc. Ser. A Math. Phys. Eng. Sci., 456 (2000), pp. 1101-1115.

[28] E. ZuAzuA, Finite dimensional null controllability for the semilinear heat equation, J. Math. Pures Appl., 76 (1997), pp. 570-594.

Copyright (c) by SIAM. Unauthorized reproduction of this article is prohibited. 